\title{
CIÊNCIANATURA
}

\section{Abordagem radiométrica/espectral na identificação de recifes de corais por meio de sensor orbital}

\author{
Radiometric/spectral approach in the identification of coral reefs by orbital sensor
}

\section{Vagner Figueiredo dos Santos ${ }^{1}$, Pablo Santana Santos ${ }^{1}$, Ruy Kenji Papa de Kikuchi ${ }^{2}$ e Danilo Heitor Caires Tinoco Bisneto Melo ${ }^{1}$}

\author{
${ }^{1}$ Universidade Federal da Bahia / Departamento de Oceanografia/ INCT AmbTropic, BA, Brasil \\ ${ }^{2}$ Universidade Federal da Bahia - Instituto de Geociências , BA, Brasil
}

\section{Resumo}

No Brasil, os recifes de coral estão distribuidos praticamente por toda a costa tropical. A urbanização das regiões costeiras e as mudanças do clima em escala global podem ocasionar impactos negativos sobre esse ecossistema, onde a identificação dos recifes de corais por sensoriamento remoto é uma iniciativa importante para facilitar a gestão dessas áreas. O presente trabalho propõe uma avaliação radiométrica/ espectral de três bancos de recifes de corais do Complexo Recifal de Abrolhos-BA (Viçosa, Coroa Vermelha e Sebastião Gomes), por meio de sensibilidade gráfica e Optimum Index factor (OIF), em imagens do satélite OLI/LANDSAT-8. A sensibilidade dos três bancos de recifes de corais supracitados foi semelhante. As bandas espectrais B1, B2, B3, B4 apresentaram sensibilidade para realçar os recifes de corais, da água do mar, sendo que a partir da região do infravermelho-próximo $(\geq B 5)$ não houve qualquer sensibilidade, mesmo para alguns recifes de corais emersos, conforme demonstrou o teste estatístico $t$ (Student), onde não houve diferença estatísticas entre os valores digitais dos recifes de corais e a água do mar, já que o Valor-p foi maior que o nivel de significância adotado (alfa =0,05). Das quatro bandas espectrais da região do visivel (B1, B2, B3 e B4), apenas a B1 não apresentou diferença significativa. Segundo OIF a melhor composição colorida para diferenciação visual dos recifes de corais da água do mar foi $1 R 2 G 4 B(5.403,3)$.

Palavras-chave: Sensoriamento Remoto. Recifes de corais. Processamento digital de imagens.

\section{Abstract}

In Brazil, the coral reefs are distributed for practically the whole tropical coast. The urbanization of coastal regions and changes of the climate in global scale can occasion negative impacts on this ecosystem, where the identification of the coral reefs from remote sensing is an important initiative to facilitate the management of these areas. This paper proposes a radiometric/spectral evaluation of three banks in the Complex Coral Reefs of the Abrolhos-BA (Viçosa, Coroa Vermelha and Sebastião Gomes), through graphic sensitivity and Optimum Index Factor (OIF), in satellite images OLI/Landsat-8. The sensitivity of the three banks in coral reefs was similar. The spectral bands B1, B2, B3, B4 showed sensitivity to enhance the coral reef, the sea water, and from the near-infrared region $(\geq B 5)$ there was no any sensitivity, even for some emerged coral reefs, as demonstrated statistical Test-t (Student), where there was no difference statistics between the digital values of coral reefs and sea water, since the p-Value was greater than the significance level (alpha $=0.05)$. Of the four spectral bands in the visible region $(B 1, B 2, B 3$ and B4), only the B1 presented no significant difference. According to OIF the best color composition for visual differentiation of sea water of coral reefs was $1 R 2 G 4 B$ (5403.3).

Keywords: Remote sensing. Coral reef. Digital image processing. 


\section{Introdução}

Os recifes de corais estão presentes em aproximadamente cem países pelos trópicos, e nos mares é um dos ecossistemas mais diversos, com alto potencial econômico interferindo direta ou indiretamente na vida de aproximadamente quinhentos milhões de pessoas ao redor do mundo (FERREIRA 2006). Ocupam uma porção inferior a 0,02\% da área de todo o oceano, entretanto, abriga $25 \%$ de todas as espécies marinhas, e estão distribuídos em regiões oceânicas tropicais, de água límpida, de baixo material em suspensão, relativamente pobre em nutrientes, e elevada incidência solar, limitando-se à zona eufótica com temperatura média anual da água de $20^{\circ} \mathrm{C}$ e de alta salinidade (BARRADAS et al., 2010; SANTOS e SALIMOS, 2016). Tais variáveis ambientais nas águas marinhas permitem a simbiose alga-coral, essencial para o recife de coral se desenvolver (KINZIE e BUDDMEYER, 1996).

São considerados como os mais complexos e produtivos dos ecossistemas marinhos (CONNEL, 1978), pois apresentam grande capacidade de reciclagem de nutrientes (LEÃO, 2009), que pode equivaler a uma produtividade de 1.500 a $5.000 \mathrm{gCm}^{-2} \mathrm{ano}^{-1}$ quando em associação a luminosidade ideal, temperatura elevada e movimentos hidrodinâmicos (SANTOS e SALIMOS, 2016).

Muitas das espécies recifais integram cadeias tróficas marinhas e costeiras (CONNELL, 1978), abrigam estoques pesqueiros e auxiliam na subsistência de várias comunidades tradicionais. Agem contra a ação erosiva hidrodinâmica das ondas, ou seja, como proteção a costa (LEÃO, 1996). São também recursos importantes para a exploração do turismo, fornecem dados científicos para análises biológicas, climáticas e geológicas (WILKINSON, 2000). Os corais são tidos como excelentes bioconstrutores de carbonato de cálcio (BARNES e DEVEREUX, 1984), e, juntamente com espécies como algas coralináceas incrustantes, gastrópodos, briozoárias, entre outras, constroem ambientes ricos em recursos naturais e de grande importância ecológica, econômica e social. Portanto, os recifes são áreas prioritárias à conservação marinha (SILVEIRA et al., 2014).

Sua distribuição, no Brasil, ocorre praticamente ao longo de toda a costa tropical, desde o Parque Estadual Marinho Parcel do Manuel Luís, no Estado do Maranhão, até as imediações da foz do Rio Doce, no Espírito Santo (LEÃO et al., 2003), associados a crostas de algas calcárias, briozoários incrustantes e outras estruturas de carbonato de cálcio de fonte orgânica (carapaças e conchas) (CORREIA e SORVIERZOSKY, 2005). Abrolhos é o Complexo Recifal mais extenso do Oceano Atlântico Sul, e seus recifes de corais têm aspectos bem definidos, distribuídos em três tipos fundamentais, que são respectivamente, arco costeio, arco externo e franja. Estão localizados entre 10 a $70 \mathrm{~km}$ da costa, podendo chegar a 25 metros de profundidade (LEÃO, 1999).

Pesquisas relacionadas à identificação desses recifes de corais são de extrema importância, pois o crescente desenvolvimento urbano em regiões costeiras (LEÃO et al., 1994), aliado às consequências diretas das mudanças globais (LEÃO et al., 2016), tem gerado forte pressão sobre estes recursos naturais. A identificação destes complexos edifícios orgânicos pode dar subsídios a diversos estudos sobre este ecossistema, fornecedor de recursos naturais, e algumas pesquisas têm utilizado imagens orbitais para mapeamento sinótico de recifes de corais com a finalidade de auxiliar no seu gerenciamento, tão importante para o desenvolvimento da vida marinha (LUBIN et al., 2000; ANDRÉFOUET et al., 2001).

Figura 1 - Localização dos três recifes de corais selecionados do Complexo Recifal de Abrolhos

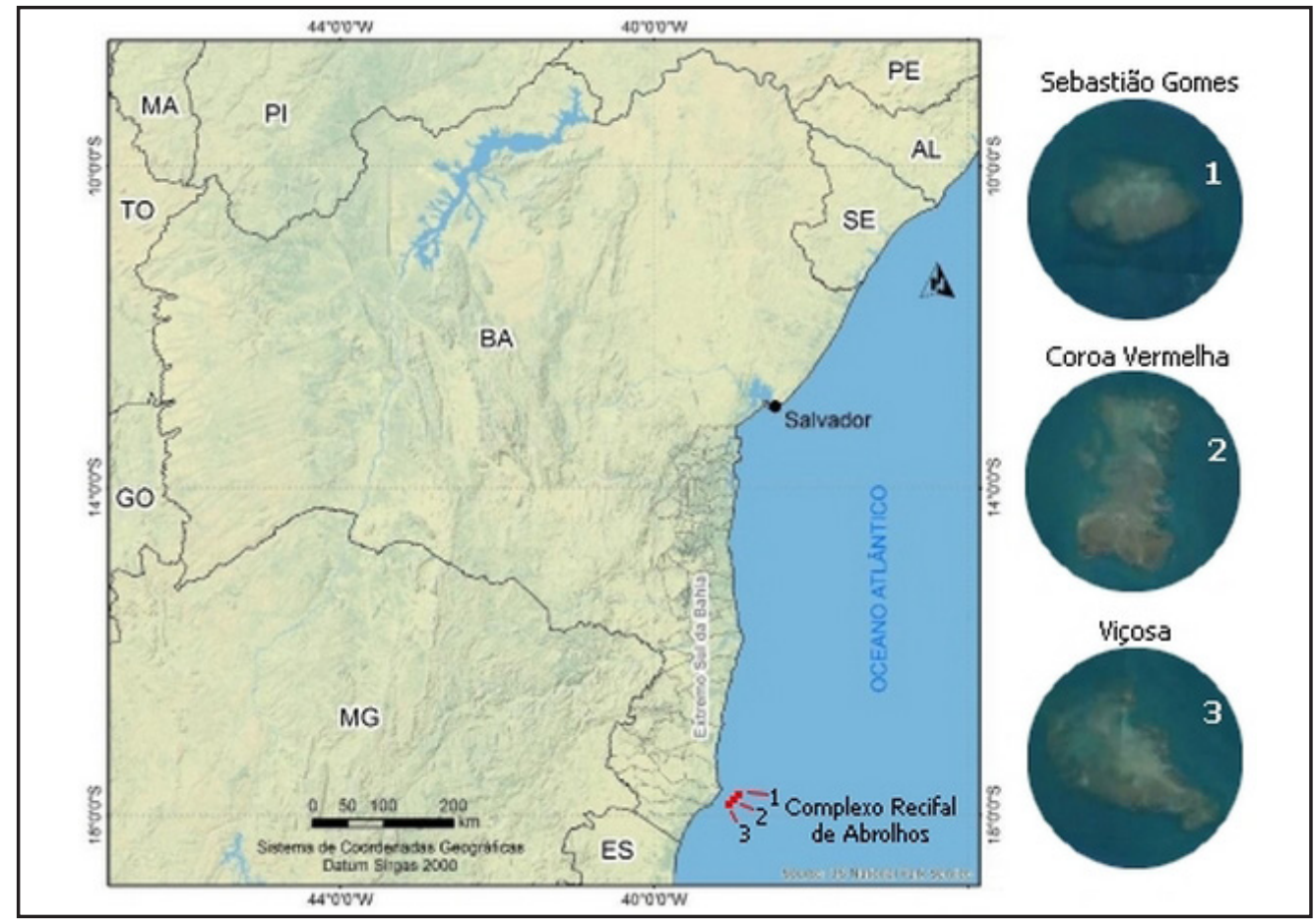


Desde o início do programa LANDSAT, programa espacial para a construção de satélites imageadores de observação terrestre, criado pela Agência Espacial Americana (NASA), houve um grande impulso nas pesquisas científicas que utilizam imagens/produtos satelitários. Recentemente, mais uma série deste programa espacial foi posto em órbita, o satélite OLI/LANDSAT-8, com melhorias, sobretudo nas abordagens espectrais e radiométricas, seja pelo aumento do número de bandas, como também na amplitude dos valores radiométricos, que passaram de $8(256 \mathrm{DN})$ para $16(65.536 \mathrm{DN})$ bits. Neste sentido, por ser um satélite relativamente novo, existem ainda poucos trabalhos que se propõem a avaliar o potencial destas imagens em detecção de recifes de corais, considerando as melhorias espectrais e radiométricas quando comparado às imagens do programa LANDSAT das séries anteriores.

Este artigo tem como objetivo principal avaliar o potencial das imagens do satélite OLI/LANDSAT-8, na identificação de bancos de recifes de corais, conhecidos do Complexo Recifal de Abrolhos (Viçosa, Coroa Vermelha e Sebastião Gomes), a partir de uma abordagem radiométrica/espectral, com o uso de gráficos de sensibilidade e Index Optimum Factor (IOF).

\section{Procedimentos metodológicos}

\subsection{Localização da área de estudo}

A área de estudo corresponde ao Complexo Recifal de Abrolhos, localizada na Mesorregião Extremo Sul da Bahia (IBGE, 2007), onde se encontra o Parque Nacional Marinho de Abrolhos, delimitada pelas seguintes coordenadas geográficas centrais: Lat. $17^{\circ}$ 44'34" S e Long. 38 49' 52" W. Nesta área encontram-se mais de 18 espécies de corais catalogadas, sendo 7 endêmicas no Brasil (MMA/SBF, 2003). Foram selecionados para a realização da pesquisa três bancos de recifes de corais, são eles: Viçosa, Coroa Vermelha e Sebastião Gomes (Figura 1).

As imagens do satélite OLI/LANDSAT-8 foram adquiridas de maneira gratuita no portal da Agência Espacial Americana (NASA), por meio do sítio http://earthexplorer.usgs.gov. Estas possuem profundidade radiométrica de 16 bits, e foram selecionadas 07 bandas espectrais monocromáticas no espectro reflexivo: B1-Azul Costeiro [0,430-0,450 $\mu \mathrm{m}]$; B2-Azul [0,450-0,510 $\mu \mathrm{m}]$; B3-Verde [0,530-

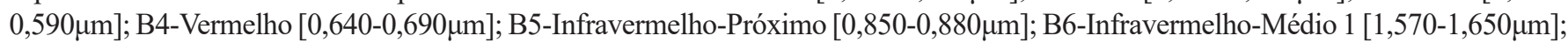

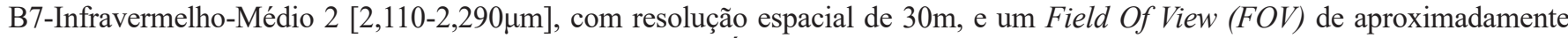
185km. Foram pré-selecionadas duas cenas posicionadas na Órbita/Ponto 215/072 (Cena 1 - 01/01/2016; Cena 2 - 21/03/2016), com horário de aquisição às 09:28 a.m., que passaram inicialmente por duas avaliações preliminares: em relação a tábua de maré no dia e horário da passagem do satélite, objetivando menor altura da lâmina d'água do mar, e uma outra avaliação referente a presença de nuvens na área de estudo, por se tratar de uma região costeira, onde as imagens são oriundas do sensoriamento remoto passivo.

A avaliação da tábua de maré foi baseada nas estimativas do sítio Tábuas de Marés (http://www.tabuademares.com), para o município litorâneo mais próximo ao Complexo Recifal de Abrolhos, Caravelas-BA. A Cena 2 com data de passagem em 21/03/2016 foi a que apresentou menor altura da tábua de maré no momento da passagem do satélite OLI/LANDSAT-8 (Figura 2), entretanto, tinha incidência de nuvens sobre os três recifes de corais selecionados para a avaliação radiométrica/espectral. Desta forma, mesmo com a maré um pouco mais alta, não houve outra alternativa senão selecionar a Cena 01, aquisição com uma boa janela atmosférica, tendo em vista os pouquíssimos registros recentes livres de nuvens desta região.

Figura 2 - Variação de maré em Caravelas - BA, onde é possível observar uma tábua de maré menor para a data 21/03/2016 (Cena 2)

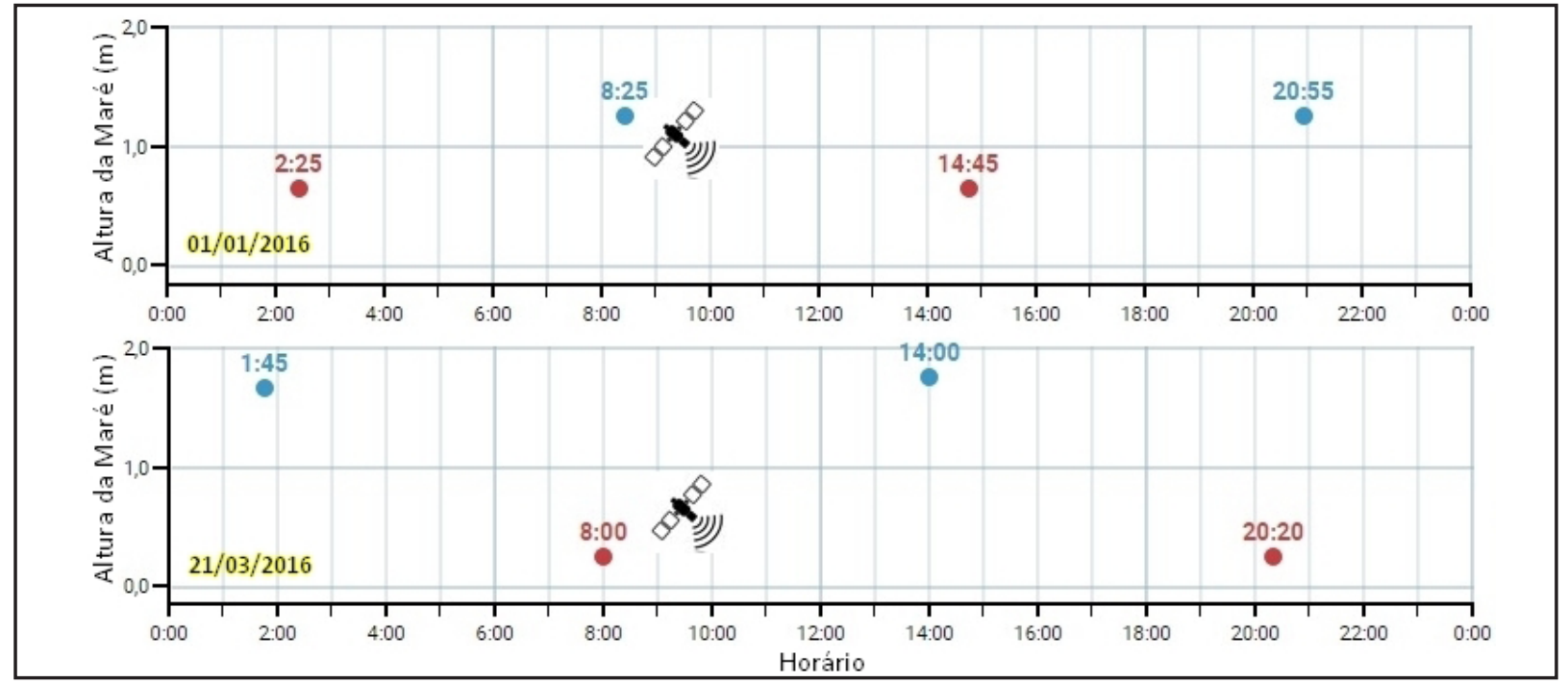

Fonte: Adaptado de Tabuas de Maré (2016) 
Após a seleção da Cena 01 (01/01/2016), foi realizada uma análise de sensibilidade radiométrica/espectral das sete bandas espectrais do satélite OLI/LANDSAT-8, similar à avaliação realizada por Santos (2014) (Figura 3), para identificar quais bandas espectrais apresentam sensibilidade que auxiliem na diferenciação dos recifes de corais dos corpos d'água (água do mar). Tal etapa foi realizada com projeção de um transecto de aproximadamente $5 \mathrm{~km}$ no sentido perpendicular ao norte geográfico, e foi realizada no software gratuito QGIS, que pode ser acessado pelo sítio http://www.qgis.org.

A fim de testar estatisticamente a similaridade entre os valores digitais da água do mar e os encontrados nos recifes de corais, utilizou-se dois testes estatísticos: Teste F (Fisher) de análise das variâncias, e Teste t (Student) de diferença/similaridade para duas amostras pareadas.

Em seguida, por meio da estatística descritiva das bandas espectrais, a partir de uma análise fatorial baseada na correlação e no desvio padrão, foi gerado o Optimum Index factor (OIF) (QAID e BASAVARAJAPPA, 2008), descrito na Equação 1, com objetivo de estabelecer um Rank, e assim identificar qual a melhor composição colorida para se trabalhar processos de interpretação visual na identificação e delimitação de recifes de corais, tendo como piloto os recifes escolhidos. Para esta etapa metodológica foi utilizado o software gratuito Ilwis, de acesso por meio do sítio http://www.ilwis.org.

$$
\mathrm{OIF}=(\sigma(\mathrm{i})+\sigma(\mathrm{j})+\sigma(\mathrm{k})) /(\mathrm{c}(\mathrm{i}, \mathrm{j})+\mathrm{c}(\mathrm{i}, \mathrm{k})+\mathrm{c}(\mathrm{j}, \mathrm{k}))
$$

Onde: $\sigma$ é o desvio padrão, c é o coeficiente de correlação, e (i), (j), (k) são as bandas espectrais.

Salienta-se que os valores radiométricos das bandas espectrais foram analisados em níveis digitais (ND), sem a correção atmosférica do espalhamento e absorção, pois objetiva-se nesta pesquisa apenas avaliar se há diferença de sensibilidade nas bandas espectrais entre a água do mar e os recifes de corais, e não medir a grandeza física do comportamento espectral destes dois alvos.

Figura 3 - Avaliação radiométrica dos valores digitais nas imagens do satélite Landsat-5

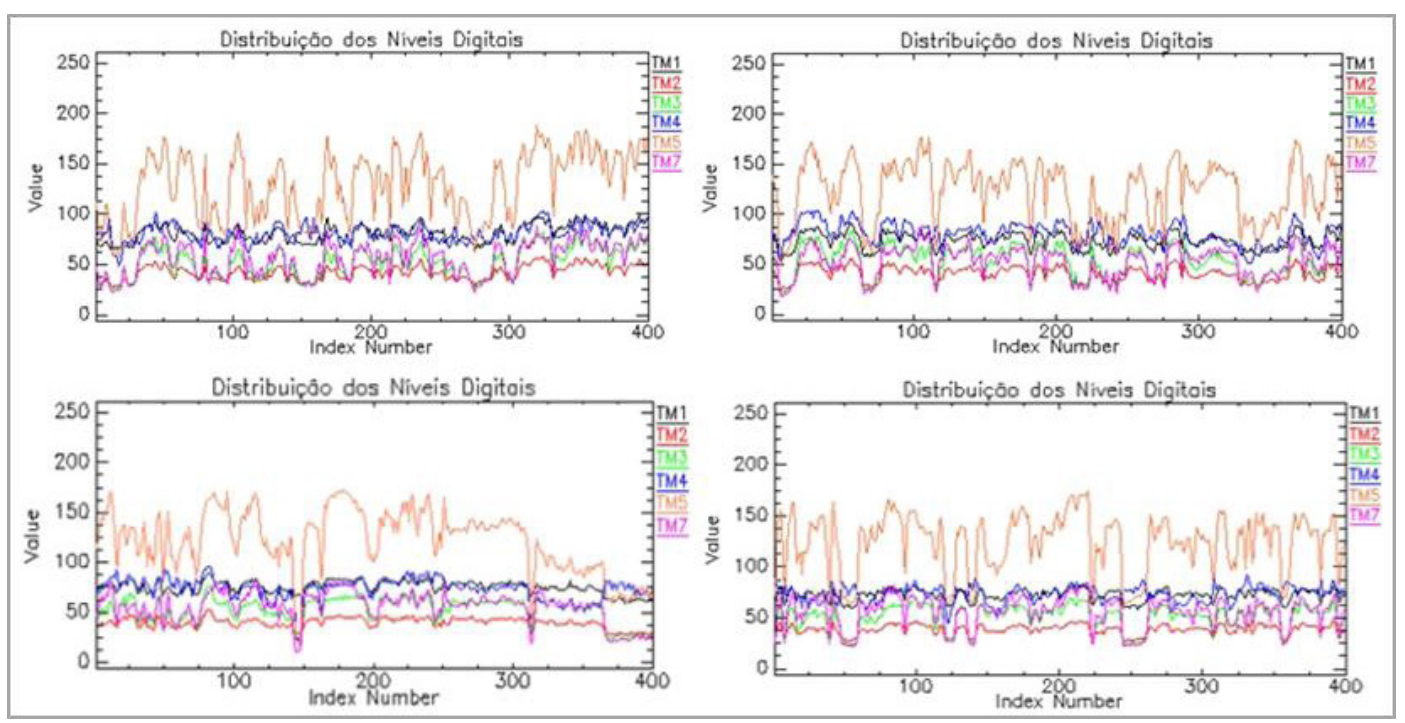

Fonte: Santos (2014)

\section{Resultados e Discussão}

A diferença de sensibilidade da água do mar e dos recifes de corais foram bem similares nas três áreas selecionados (Viçosa, Coroa Vermelha e Sebastião Gomes). As bandas da região do visível (B1-Azul Costeiro [0,430-0,450 $\mu$ m]; B2-Azul [0,450-

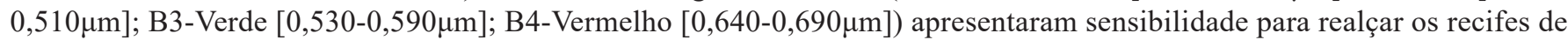
corais da água do mar, e esta distinção se eleva à medida que se aumenta o tamanho do comprimento de onda, de modo que a banda espectral B4-Vermelho $[0,640-0,690 \mu \mathrm{m}]$ foi a que apresentou maior amplitude de distinção. Por outro lado, a partir do

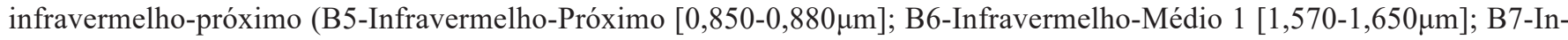

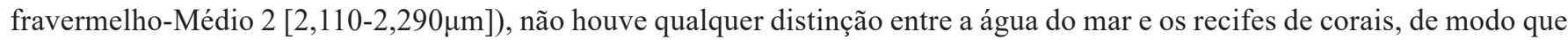
tais bandas espectrais podem ser completamente descartadas para tal aplicação (Figura 4).

É sabido que a radiação eletromagnética (REM) tem elevada absorção e baixa reflectância ( 4\%) quando interage com a água límpida em estado líquido, a partir da faixa espectral do infravermelho-próximo, assim como evidenciam os diversos trabalhos de sensoriamento remoto do comportamento espectral da água (NOVO, 2002; JENSEN, 2009). Entretanto, como há 
em alguns casos organismos (corais, algas e zoantídeos) emersos, e outros a uma baixa profundidade, esperava-se uma diferença de sensibilidade, mesmo que mínima. Em função dos valores encontrados, verifica-se que superfícies úmidas não apresentam qualquer diferenciação nas bandas espectrais do OLI/LANDSAT-8 a partir do infravermelho-próximo.

4 - Análise de sensibilidade de distinção entre a água do mar e os recifes de corais

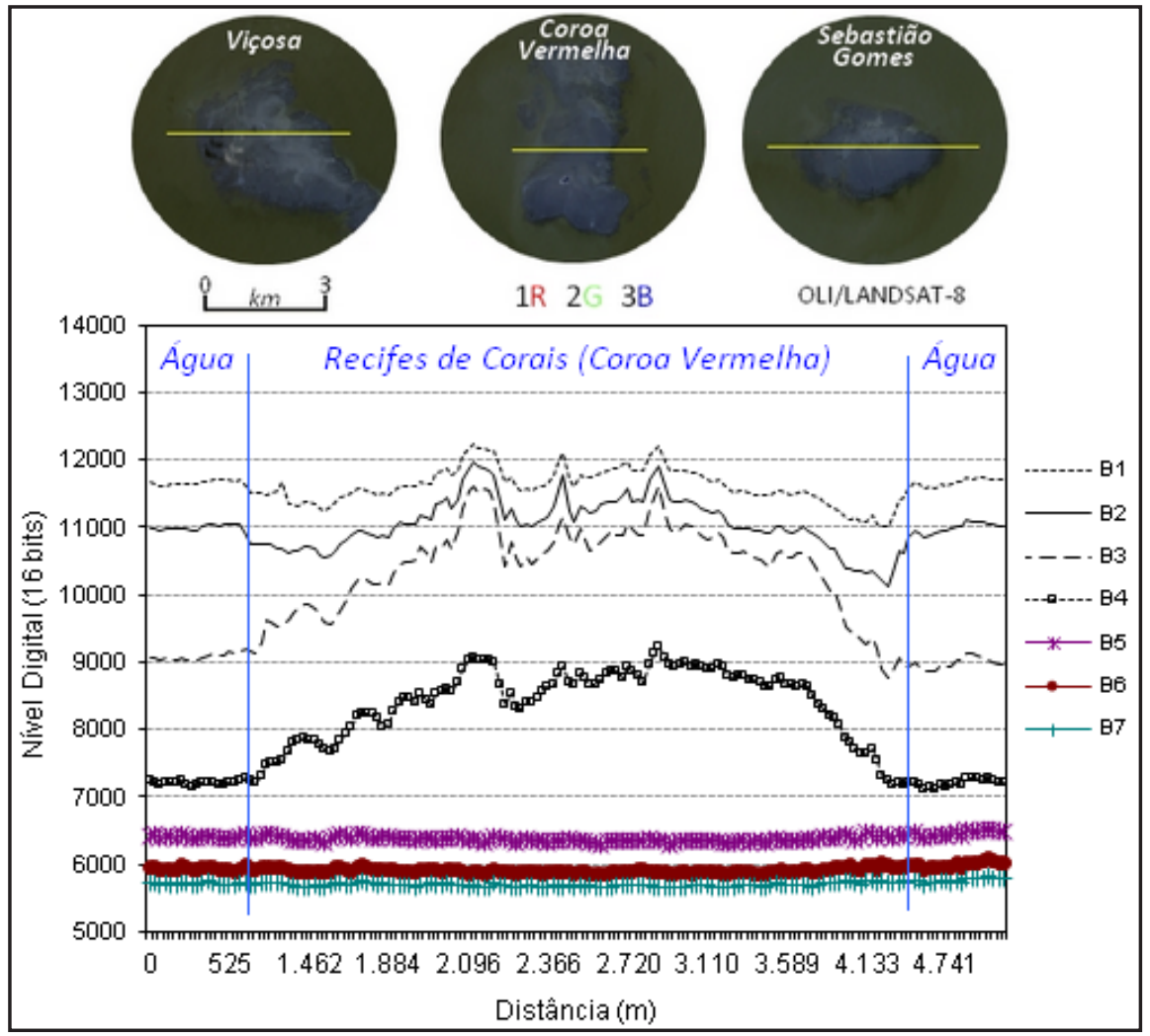

Os testes estatísticos abaixo são representados pela similaridade/distinção entre os valores médios digitais encontrados nas 60 amostras sobre o transecto nos três recifes de corais, com a metade representando os valores digitais da Água do Mar (A), e a outra metade quantifica os valores digitais referentes aos recifes de corais (RC). O Teste $\mathrm{F}$ (Fisher) demostrou que apenas as

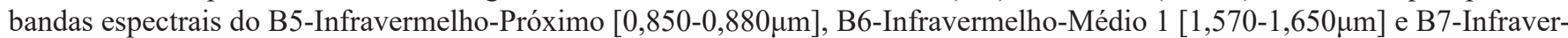

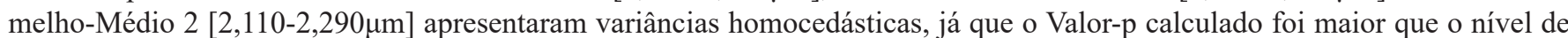
significância adotado (alfa $=5 \%$ ). Desta forma, o Teste $\mathrm{t}($ Student $)$ foi condicionado especificamente para estas bandas espectrais sobre a regra de admitir variâncias iguais entre A e RC, para todas as bandas espectrais as variâncias foram heterocedásticas.

Tabela 1. Resultado dos testes estatísticos (Fisher e Studant) para as 60 amostras, 30 água do mar (A), e 30 de recifes de corais (RC)

\begin{tabular}{l|c|c|c|c|c|c|c}
\hline Teste F (Fisher) & $\mathrm{B} 1(\mathrm{~A} / \mathrm{RC})$ & $\mathrm{B} 2(\mathrm{~A} / \mathrm{RC})$ & $\mathrm{B} 3(\mathrm{~A} / \mathrm{RC})$ & $\mathrm{B} 4(\mathrm{~A} / \mathrm{RC})$ & $\mathrm{B} 5(\mathrm{~A} / \mathrm{RC})$ & $\mathrm{B} 6(\mathrm{~A} / \mathrm{RC})$ & $\mathrm{B} 7(\mathrm{~A} / \mathrm{RC})$ \\
\hline \hline Razão & 0,293 & 0,208 & 0,308 & 0,373 & 0,740 & 0,978 & 1,019 \\
\hline F (Valor observado) & 0,293 & 0,208 & 0,308 & 0,373 & 0,740 & 0,978 & 1,019 \\
\hline F (Valor crítico) & 2,101 & 2,101 & 2,101 & 2,101 & 2,101 & 2,101 & 2,101 \\
\hline GL & 29 & 29 & 29 & 29 & 29 & 29 & 29 \\
\hline Valor-p (bilateral) & 0,001 & $<0,0001$ & 0,002 & 0,010 & 0,422 & 0,952 & 0,961 \\
\hline alfa & 0,05 & 0,05 & 0,05 & 0,05 & 0,05 & 0,05 & 0,05 \\
\hline Teste t (Student) & $\mathrm{B} 1(\mathrm{~A} / \mathrm{RC})$ & $\mathrm{B} 2(\mathrm{~A} / \mathrm{RC})$ & $\mathrm{B} 3(\mathrm{~A} / \mathrm{RC})$ & $\mathrm{B} 4(\mathrm{~A} / \mathrm{RC})$ & $\mathrm{B} 5(\mathrm{~A} / \mathrm{RC})$ & $\mathrm{B} 6(\mathrm{~A} / \mathrm{RC})$ & $\mathrm{B} 7(\mathrm{~A} / \mathrm{RC})$ \\
\hline Diferença & $-12,033$ & $-142,033$ & $-1089,767$ & $-930,233$ & 13,267 & 13,733 & 6,367 \\
\hline $\mathrm{t}$ (Valor observado) & $-0,266$ & $-2,177$ & $-10,790$ & $-12,375$ & 1,747 & 1,975 & 1,212 \\
\hline$|\mathrm{t}|$ (Valor crítico) & 2,015 & 2,020 & 2,014 & 2,011 & 2,002 & 2,002 & 2,002 \\
\hline GL & 58 & 58 & 58 & 58 & 58 & 58 & 58 \\
\hline Valor-p (bilateral) & 0,791 & 0,035 & $<0,0001$ & $<0,0001$ & 0,086 & 0,053 & 0,231 \\
\hline alfa & 0,05 & 0,05 & 0,05 & 0,05 & 0,05 & 0,05 & 0,05 \\
\hline
\end{tabular}




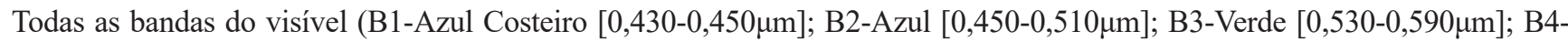
Vermelho $[0,640-0,690 \mu \mathrm{m}])$ foram submetidas a análise Optimum Index factor $(O I F)$, de modo que a melhor composição colorida indicada pelo índice foi 1R 2G 4B (5.403,3), ou seja, esta composição colorida falsa-cor foi a primeira do Rank (Figura 5) para facilitar processos de interpretação visual na identificação de recifes de corais utilizando imagens orbitais do satélite OLI/LANDSAT-8.

Figura 5 - Indicação, pelo Optimum Index factor (5.403,3), da melhor composição colorida para facilitar a aplicação de metodologias de classificação visual

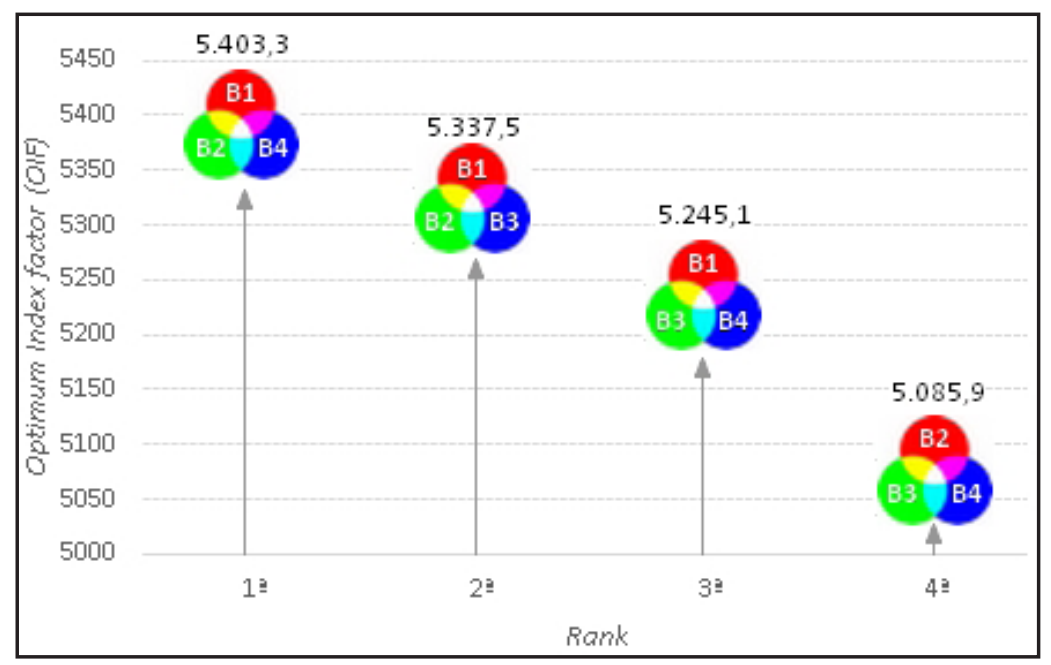

A figura abaixo (Figura 6) mostra que não há qualquer possibilidade de uso de bandas espectrais a partir do infravermelho-próximo para identificar esses ecossistemas, os recifes de corais são indetectáveis visualmente nas composições coloridas a partir deste faixa espectral. Já ao usar a composição colorida falsa-cor 1R 2G 4B indicada pelo OIF (5.403,3) facilmente esses recifes de corais são identificados visualmente nas imagens do satélite OLI/LANDSAT-8. Portanto, ao usar este sensor orbital aplicado a identificação de recifes de corais por processos de classificação visual, recomenda-se utilizar a composição colorida supracitada.

Figura 6 - Comparação entre a composição colorida com as bandas a partir do infravermelho-médio, onde não é possível detectar os recifes de corais por interpretação visual, com a composição colorida indicada pelo OIF

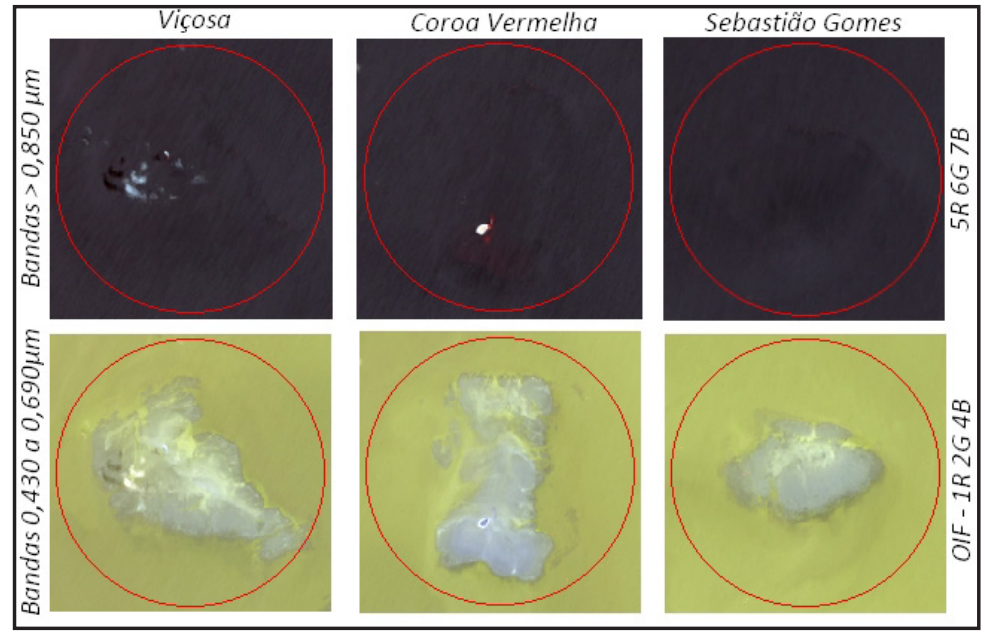

\section{Conclusões}

É possível realizar a distinção dos recifes de corais da água do mar com base nas imagens do satélite OLI/LANDSAT-8, utilizando algumas bandas espectrais. Os três recifes de corais apresentaram padrões similares, sendo que as análises de sensibilidade não apresentaram qualquer distinção que auxiliem na identificação dos recifes de corais a partir da região do infravermelho-próximo, mesmo em alguns corais emersos. A condição de umidade mesmo com o objeto situado acima do nível do mar (emersos) tem contribuição definitiva para tal resultado. 
Santos et al. : Abordagem radiométrica/espectral na identificação de recifes de corais por meio de sensor orbital

As bandas do visível (B1, B2, B3 e B4) graficamente apresentaram sensibilidade de distinção, entretanto, é importante ressaltar que a B1 (Azul Costeiro) não alcançaram significância estatística. Segundo análise do Optimum Index factor (OIF) a melhor composição colorida para facilitar processos visuais de identificação dos recifes de corais foi 1R 2G 4B $(5.403,3)$.

\section{Agradecimentos}

Agradecemos ao Programa Permanecer da Universidade Federal da Bahia, pela concessão da bolsa de estudo, fundamental para o desenvolvimento da pesquisa. RKPK é bolsita $1 \mathrm{C}$ do CNPq. Este artigo é uma contribuição do Instituto Nacional de Ciência e Tecnologia Ambientes Marinhos Tropicais (INCT AmbTropic).

\section{Referências}

ANDRÉFOUET, S.; MULLER-KARGER, F. E.; HOCHBERG, E. J.; HU, C.; CARDER, K. L. Change detection in shallow coral reef environments using Landsat 7 ETM+ data. Remote Sensing of Environment. 2001;78(1): 150- 162.

BARNES, D. J.; DEVEREUX, M. J. Productivity and calcification on a coral reef: a survey using $\mathrm{pH}$ and oxigen electrode techniques. Journal of Experimental Marine Biology and Ecology. 1984;79(3): 213-231.

BARRADAS, J. I.; AMARAL, F. D.; HERNÁNDEZ, M. I. M.; FLORES-MONTES, M. J.; STEINER, A. Q. Spatial distribution of benthic macroorganisms on reef flats at Porto de Galinhas Beach (northeastern Brazil), with special focus on corals and calcified hydroids. Revista Biotemas. 2010;23(2): 61-67.

CONNELL, J. H. Diversity in tropical rain forest and coral reefs. Science. 1978;199(4335): 1302- 1310.

CORREIA, M. D.; SOVIERZOSKI, H. H. Ecossistemas marinhos: recifes, praias e manguezais. 1st ed. Maceió: EDUFAL; 2005.

FERREIRA, P. B.; MAIDA, M. Monitoramento dos recifes de coral do Brasil. 1st ed. Brasília: MMA; 2006.

IBGE, Instituto Brasileiro de Geografia e Estatística: Mapeamento das unidades territoriais. Brasília (Brasil): Ministério do Planejamento, Desenvolvimento e Gestão, 2007.

JENSEN, J. R. Sensoriamento remoto do ambiente: uma perspectiva em recursos terrestres. 1st Engl. ed. Epiphanio, J.C.N., tradução. São José dos Campos: Parêntese; 2009. 598 p.

KINZIE, R. A.; BUDDEMEIER, R. W. Reefs happen. Global Change Biology. 1996;2(6): 479-494.

LEÃO, Z. M. A. N. Abrolhos: O complexo recifal mais extenso do Oceano Atlântico Sul. Sítios Geológicos e Paleontológicos do Brasil. 1st ed. Brasília: SIGEP; 1999.

LEÃO, Z. M. A. N. The coral reefs of Bahia: morphology, distribution and the major environmental impacts. Anais da Academia Brasileira de Ciências. 1996;68(3): 439-452.

LEÃO, Z. M. A. N. KIKUCHI, R.K.P., FERREIRA, B.P., NEVES, E.G., SORVIERZOSKI, H.H., OLIVEIRA, M.D.M., MAIDA, M., CORREIRA, M.D., JOHNSSON, R. Brazilian coral reefs in a time of global changes: a synthesis. Brazilian Journal of Oceanography. 2016;64(2): 97-115.

LEÃO, Z.M.A.N., TELLES, M.D., SFORZA, R., BULHÕES, H.A., KIKUCHI, R.K.P. Impacts of tourism development on the coral reefs of the Abrolhos area, Brazil. 1994, Miami. Anais... Miami: RSMAS, 1994. p. 254-260.

LEÃO, Z. M. A. N., KIKUCHI, R. K. P., AMARAL, F. D, OLIVEIRA, M. D. M.; COSTA, C. F. Recifes de Corais-Tesouros agonizantes. Scientific American Brasil (Edição Especial). 2009;Oceano: 74-82.

LEÃO, Z. M. A. N.; KIKUCHI, R. K. P.; TESTA, V. Corals and coral reefs of Brazil. In: Cortés, J., editors. Latin American coral reefs. Amsterdam: Elsevier Science; 2003. p. 9-52. 
LUBIN, D.; LI W.; DUSTA, P.; MAZEL, C. H. L, STAMNES, K. Spectral signatures of coral reefs: features from space. Remote Sensing of Environment. 2000;75(1): 129- 137.

MMA/SBF, Ministério do Meio Ambiente/Secretaria de Biodiversidade e Florestas: Atlas dos Recifes de Coral nas Unidades de Conservação Brasileiras. Brasília (Brasil): Ministério do Meio Ambiente, 2003. 232p.

NOVO, E. M. L. Sensoriamento Remoto, Principios e Aplicações. 2st ed. São Paulo: Edgar Blucher Ltda; 2002.

QAID, A. M.; BASAVARAJAPPA, H. T. Application of optimum index factor technique to Landsat-7 data for geological mapping of north east of Hajjah, Yemen. American-Eurasian Journal of Scientific Research. 2008;3(1): 84-91.

SANTOS, P. S. Caracterização e mapeamento biofísico ambiental da bacia hidrográfica do rio Vermelho a partir de dados remotamente sensoriados [thesis]. Goiânia: Instituto de Estudos Sócio-Ambientais/UFG; 2014. 157 p.

SANTOS, T.; SALIMOS, F. Produção primária média da costa leste australiana no período de janeiro de 2011 a janeiro de 2012 por imagens do sensor MODIS. Boletim do Laboratório de Hidrobiologia. 2016;26(1): 26-31.

SILVEIRA, C. B. L.; FERREIRA, B. P.; COXEY, M. S. Variação temporal nos recifes de corais de Maragogi, APA Costa dos Corais (2010 - 2013). Tropical Oceanography. 2014;42(2): 208-225.

WILKINSON, C. R. Worldwide coral reef bleaching and mortality during 1998: A global climate change warming for the new millennium? Seas at the Millennium, an environmental evaluation. 2000;3(1): 43-57.

Vagner Figueiredo dos Santos
Instituto de Geociências da Universidade Federal da Bahia, BA, Brasil
Email: vagner147vfs@gmail.com

\section{Pablo Santana Santos}

Instituto de Geociências da Universidade Federal da Bahia, BA, Brasil Email: pablo.srgeo@gmail.com

Participação do autor: Contribuições substanciais para a concepção do trabalho; Aquisição, análise ou interpretação de dados.

\footnotetext{
Ruy Kenji Papa de Kikuchi

Universidade Federal da Bahia - Departamento de Oceanografia/ INCT AmbTropic, BA, Brasil Email:kikuchi@ufba.br

Participação do autor: Contribuições substanciais para a concepção do trabalho; Realizou uma revisão substancial
}

\footnotetext{
Danilo Heitor Caires Tinoco Bisneto Melo

Universidade Federal da Bahia - Instituto de Geociências , BA, Brasil Email: danilohmelo@gmail.com

Participação do autor: Contribuições substanciais para a concepção do trabalho; Aquisição, análise ou interpretação de dados.
} 